\title{
Analysis on Stem cell Treatment of Alzheimer's Disease
}

\author{
Weixun $\mathrm{Zhu}^{1 *}$ \\ ${ }^{1}$ School of Life Science, Central South University, 410006 Changsha, China
}

\begin{abstract}
Alzheimer's disease is a neurodegenerative disease characterized by progressive memory loss and cognitive impairment. At present, conventional drugs have little effect on the prevention and treatment of $\mathrm{AD}$, and the rapidly developing stem cell transplantation therapy provides a new direction for the treatment of neurodegenerative diseases. In this paper, we can conclude that stem cell therapies such as neural stem cells and mesenchymal stem cells have shown curative effects in the treatment of neurodegenerative diseases such as Alzheimer's disease by analyzing existing stem cell research and analysis at this stage, and there will be huge applications in the future. prospect. Stem cells can secrete immunoregulatory factors through paracrine pathways to inhibit inflammation and reduce the accumulation of amyloid. Secrete neurotrophic factors to protect and promote nerve growth. In addition, stem cells can differentiate into neurons after transplantation, replacing damaged and dead nerve cells, and play an important role in rebuilding brain neural circuits and networks. This article analyzes and summarizes the possible ways of stem cell transplantation to treat Alzheimer's disease, which can provide new ideas and methods for future stem cell transplantation therapies.
\end{abstract}

\section{Introduction}

Population aging is one of the most prominent population problems in this century, with the incidence of some common diseases rising sharply in the elderly population, among which the incidence of Alzheimer's disease increases exponentially with age, and the elderly over 65 years old are the high incidence group of $\mathrm{AD}$ [1]. Alzheimer's disease, characterized mainly by memory and cognitive dysfunction, is a common central neurodegenerative disease. It is a serious threat to human health and development. With the increasingly serious aging, it is estimated that there will be 1 billion $\mathrm{AD}$ patients worldwide by 2030 [2].

In recent years, the research on the pathogenesis and treatment of $\mathrm{AD}$ has become a focus of attention. However, the pathogenesis of AD has not been clarified so far, and there is an extreme lack of effective treatment means. The existing treatment means are not effective, so a feasible treatment method for AD is urgently needed clinically. With the rapid development of biomedicine in recent years, relevant research has found that stem cells have the potential of self-renewal and high differentiation, which provides a new treatment strategy for neurodegenerative diseases, stem cell therapy. This paper summarizes the current reports of more stem cell therapy, and on the future of stem cell therapy in Alzheimer's disease and other neurodegenerative diseases. It will analyze and discuss the pathogenesis of $\mathrm{AD}$, the research of neural stem cells and mesenchymal stem cell transplantation for $\mathrm{AD}$, as well as the challenges and prospects in the research process.

* Corresponding author: 846010869@qq.com

\section{Pathogenesis and current drug therapy of $A D$}

\subsection{The pathogenesis of AD}

The pathophysiological mechanism of AD is complicated, and its pathogenesis has not yet been clarified. There are many factors causing $\mathrm{AD}$, such as heredity, environment, aging, metabolism. Its pathological characteristics mainly extracellular beta-amyloid (Amy - loid beta protein, A beta) deposition and age spots (senile plaques, SP), excessive phosphorylated Tau protein in cells of neurofibrillary tangles (neurofibrillary tangle, NFT) [3-4].

\subsubsection{Theory of $A \beta$ deposition and toxicity}

The traditional view is that the deposition of $A \beta$ protein in the brain is the core factor of $\mathrm{AD}$. $\mathrm{A} \beta$ protein is produced by hydrolysis of the precursor protein APP. Normally, there are two main ways to prevent A $\beta$ accumulation in the body. The first way is that the produced $A B$ is hydrolyzed by an extracellular protease and then transported out of the brain across the blood-brain barrier. The second way is to clear A $\beta$ through receptor-mediated endocytosis. However, when the mechanism of producing and removing $A \beta$ protein in the body is out of balance, A $\beta$ protein may accumulate excessively in the brain to form senile 
plaques. Because A $\beta$ protein has neurotoxicity, it can cause intracellular $\mathrm{Ca} 2+$ overload, inflammation injury, Tau protein hyperphosphorylation, synapse damage and even neuronal apoptosis. A $\beta$ also participates in mediating nerve damage by inducing chronic inflammation, which is also an important part of A $\beta$ neurotoxicity [4-5]. The accumulation of $A \beta$ easily triggers synaptic dysfunction, which causes the loss of a large number of neurons in the brain, which in turn causes dementia. Therefore, the generation of senile plaques will lead to a large number of neuronal apoptosis and atrophy of brain tissue, which in turn leads to patients with progressive memory loss and cognitive dysfunction. Therefore, inhibiting the accumulation of A $\beta$ protein and reducing its neurotoxicity can effectively prevent and treat Alzheimer's disease.

\subsubsection{Tau protein hyperphosphorylation}

According to the observation of pathological characteristics, a large number of the neurofibrillary tangle (NFT) in the cell is also one of the causes of AD, which is mainly related to the hyperphosphorylation of Tau protein. Tau protein is a kind of microtubule-associated protein, which can combine with microtubules to improve the stability of microtubules. Mainly existing in neuron axons, it plays a key role in neuron growth, material transportation in neuron cells and axon information transmission. However, with the occurrence of diseases, the physiological balance between phosphorylation and dephosphorylation of Tau protein is broken. Hyperphosphorylation of Tau protein leads to changes in the conformation of Tau protein, which in turn reduces its ability to participate in the process of microtubule assembly, and Tau protein occurs. Accumulation leads to a large number of the neurofibrillary tangle (neurofibrillary tangle, NFT), which damages nerve cells [6]. With the development of $\mathrm{AD}$, NFT continues to increase, which has led to the aggravation of AD. Studies have shown that the phosphorylation content of Tau protein in the cerebrospinal fluid of AD patients is about three times higher than that of normal people [7].

The phosphorylation state of Tau protein is mainly related to the co-regulation of a variety of protein kinases and protein phosphatases. Protein kinases mainly include three types, proline-directed protein kinases (proline-directed protein kinases, PDPKs), non-PDPKs protein kinases, and tyrosine protein kinases (tyrosine protein kinases, TPK) [8]. The main protein phosphatases include phosphoprotein phos-phatases (PPs) and phosphotyrosine phosphatases (PTPs). Studies have found that there are combinations of various protein kinases in the brains of AD patients that are involved in the phosphorylation of Tau protein. In addition, there are other phosphatases that affect the hyperphosphorylation of tau protein, and tau protein is subject to other post-translational modifications in addition to phosphorylation such as Tau acetylation[4]. In the pathological process, every step cannot be ignored.
Because tau phosphorylation is regulated by many enzymes, and the exact role of many modifications is still unclear, it is difficult to ascertain the mechanism of tau phosphorylation, leading to the unsatisfactory effect of tau protein phosphorylation in the treatment of $\mathrm{AD}$.

In addition, the mechanism of $\mathrm{AD}$ also includes the cholinergic hypothesis. The first clinical medication for AD approved by the FDA is aimed at this mechanism. The inflammatory response theory is also an important mechanism of $\mathrm{AD}$, and it is believed that the progression of $\mathrm{AD}$ is closely related to the neuroinflammatory response. At this stage, there is more in-depth research such as autophagy mechanism. [3, 4].

\subsection{Mainstream therapies and treatment bottlenecks}

Although there have been long-term studies on the treatment of $\mathrm{AD}$, no effective treatment has been found. At present, the US Food and Drug Administration (FDA) has approved the treatment by inhibiting neuronal death related proteins and restoring the function of the cholinergic neuronal system to slowly recover. The cholinergic neuron system functions in the way of treatment, so as to achieve the purpose of temporarily delaying the pathogenesis of AD. The drugs currently used for $\mathrm{AD}$ treatment mostly target a single target or only one pathogenic mechanism. For example, the earliest approved treatment for AD drug-Tacrine, is the first-generation acetylcholinesterase inhibitor ( Ach EI), but it has strong hepatotoxicity and gastrointestinal side effects, so it has been suspended clinically. Such drugs can be used to improve the cognitive level of patients with mild to moderate $\mathrm{AD}$ by inhibiting the activity of cholinesterase in the brain and promoting the release of acetylcholine in the brain [4]. Memantine, the first-choice drug for the treatment of moderate to severe AD patients, is the first N-methyl-D-aspartate receptor (NMDA) antagonist approved by the FDA. This drug antagonizes the brain of $\mathrm{AD}$ patients Abnormal hyperactivity of the excitatory transmitter glutamate can effectively inhibit the excessive activation of NMDA receptors. It is currently used to slow down the memory impairment of patients, but it has no obvious alleviating effect on the process of $\mathrm{AD}$, and it may also induce epilepsy. Severe side effects makes the use of this drug in clinical treatment very limited [9].

In recent years, as the pathogenic mechanism continues to be studied, there are other therapeutic directions in AD treatments, such as the development of anti-amyloid (beta-amyloid, A B ) drugs and antineurofibrillary tangles (NFT) durgs for the two main mechanisms of AD.It is hoped to bring more effective therapeutic drugs to AD treatment in the future.

It can be seen that by analyzing the drugs that have been used in the clinical treatment of AD and the types of drugs under development, it seems that they can only help improve the quality of life of AD patients, and have no significant effect on the occurrence and progression of the disease. There is no way to fundamentally reverse the condition of Alzheimer's disease. Moreover, the side 
effects of some drugs have a greater impact on the use of drugs. Therefore, at present, there is no specific medicine for the treatment of AD. There is an urgent clinical need for drugs and treatment methods based on the etiology of the disease to solve the rapidly developing Alzheimer's disease.

\section{Stem Cell Therapy}

Because the above-mentioned causes of AD and accelerating the course of the disease are jointly affected by many factors, plus the original $A \beta$ deposition and the hyperphosphorylation of Tau protein caused by neurofibrillary tangle (neurofibrillary tangle, NFT) are neurotoxic and will Irreversible damage to nerve cells, so the direction of $\mathrm{AD}$ treatment should focus on the early stage of onset. Effective interventions for AD before a large number of neurons degenerate and before the patient has obvious symptoms of dementia can not only curb the rapid progression of $\mathrm{AD}$ but also protect the memory and cognitive function of the patient to the greatest extent.

Since the discovery of human embryonic stem cells, the self-renewal and high differentiation potential of stem cells have opened up new ideas and approaches for the treatment of Alzheimer's disease. Stem cells may differentiate into different types of nerve cells and glial cells under artificial induction and intervention. This ability has attracted wide attention from scientists [10]. Stem cell therapy provides a high potential therapeutic method to reverse or replace some of the nerve cells lost during AD. Stem cell therapy has shown positive results such as enhancing the vitality of neurons in the brain in animal studies and has demonstrated the potential and prospects of stem cells to treat neurodegenerative diseases such as AD.

\subsection{Current status of stem cell therapy}

The potential of stem cell therapy in AD is reflected in two aspects: Firstly, existing studies have shown that stem cells can be connected to the neural network in the brain, mainly by secreting a variety of factors to regulate neurogenesis and neuroplasticity. Secondly, it may also increase the level of acetylcholine, which improves the memory and cognitive functions of $\mathrm{AD}$ animal models [11]. The main mode of action of stem cell therapy can be divided into endogenous and exogenous according to the mechanism of action. Because of the death of different types of nerve cells during the development of $\mathrm{AD}$, it is not feasible to transplant specific mature nerve cell types as a treatment for AD.Therefore, researchers pay more attention to the paracrine effect of stem cells to stimulate endogenous repair. The concept of the microenvironment is very important for the occurrence and development of the disease. For stem cell transplantation, the transplanted stem cells can secrete through paracrine way neurotrophic factor, nerve growth factor and insulin growth factor and vascular endothelial growth factor to improve the microenvironment, and the nutrient media promoted the damaged nerve cells to survive and to Alzheimer's disease brain nerve injury repair of the damaged area is of great significance [12-13].

At present, stem cell sources used in Alzheimer's disease research mainly include tissue-specific stem cells, such as neural stem cells and bone marrow mesenchymal stem cells, as well as embryonic stem cells and induced pluripotent stem cells. Neural stem cells and mesenchymal stem cells are two kinds of stem cell treatment methods that have been studied in the field of neurodegenerative diseases in recent years due to their good characteristics. The following mainly introduces the current stage of neural stem cells and mesenchymal stem cells in Alzheimer The research on the treatment of the disease and the challenges faced in the current research process.

\subsection{Application of neural stem cells in Alzheimer's disease research}

At present, neural stem cells mainly affect Alzheimer's disease in two ways: endogenous and exogenous intervention. The main difference between the two methods is that the endogenous pathway is embodied in the induction of differentiation of neural stem cells in the body so that the damaged nerve cells in the body can be repaired and regenerated. The exogenous approach is to replace damaged nerve cells in the body by transplanting neural stem cells. Neural stem cells are relatively easy to obtain and can differentiate into a variety of neural cells. Neural stem cells can differentiate into a variety of cell types including neurons, astrocytes and oligodendrocytes. Neural stem cells can be isolated from the brain tissues of fetuses and newborns after death [11]. In the animal model of $\mathrm{AD}$, it is found that the transplanted neural stem cells are widely distributed in the transplanted area and retain their original characteristics, and then can be integrated into the functional neurons of the brain to achieve effective neuron replacement. The transplanted neural stem cells are Can differentiate into mature brain cells. Moreover, it is found that neural stem cells have obvious paracrine effects after transplantation. The neurotrophic factors produced through paracrine are essential to improve the cognitive function of Alzheimer's disease. 


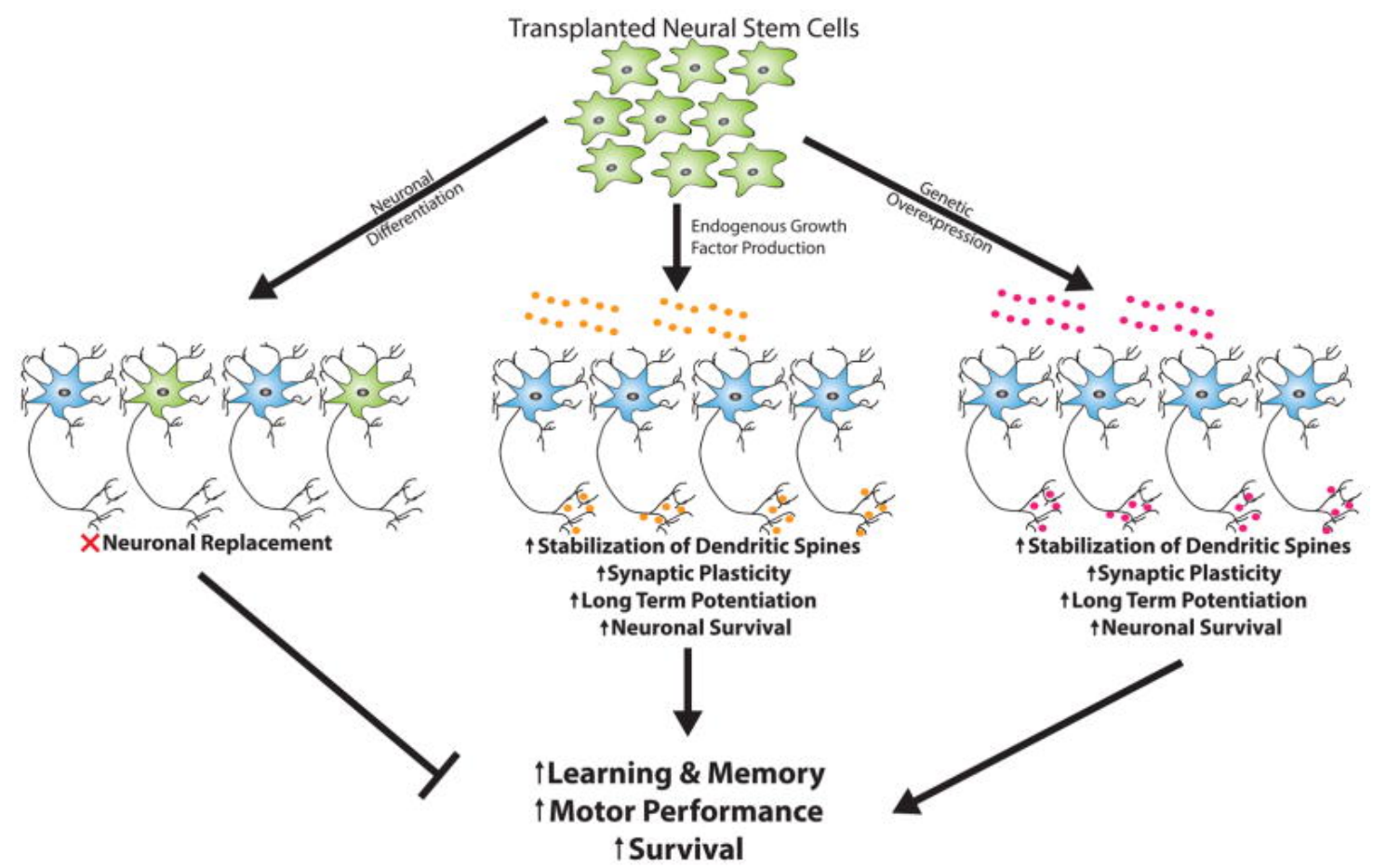

Fig. 1. Potential mechanisms of neural stem cell treatment of Alzheimer's disease.

Initially, many studies wanted to use neural stem cells to differentiate into neurons, so that the differentiated neurons could be integrated into the host neuron circuit (blue) to replace the neurons that died during $\mathrm{AD}$ to treat AD (left), but this direction Research has found huge challenges. However, recent studies have found that neurotrophic factors (middle, orange) and gene overexpression (right, purple) secreted by the endogenous pathway of neural stem cells have a good performance in the treatment of $\mathrm{AD}$ with neural stem cells and have great prospects.[20].

However, the current research and application of neural stem cells for $\mathrm{AD}$ effects still have some difficulties: first, neural stem cells undergo differentiation and other processes in the human environment, so affected by the internal environment, can neural stem cells eventually differentiate into specific functional neural cells It is not clear, and there is no clear research on the survival time of neural stem cells after differentiation, and the effectiveness and durability of neuron replacement [13-14]. However, it is gratifying that because neural stem cells can differentiate into a variety of nervous system cells, they have great prospects for cell replacement therapy; and neural stem cells show obvious paracrine effects after transplantation, which is useful for improving Alzheimer's cognitive function of patients with the disease has great potential. Moreover, the characteristics of neural stem cell transplantation due to specific neuroprotection, nerve regeneration, and immune regulation, and more importantly, the immune rejection reaction of the body produced by neural stem cell transplantation, and good fusion with host cells, all show that neural stem cells are good material for treatment. In view of the above characteristics of neural stem cells, scientists are now beginning to optimize the methods of neural stem cell therapy, such as studying neural stem cells as a carrier tool for carrying drugs, which can carry drugs currently used in clinical treatment to improve the protection of nerves. It is not just to use its neuron replacement therapy [13]. Such an approach can make the future treatment of $\mathrm{AD}$ aim to solve multiple pathogenic mechanisms so that the drug can exert a greater effect on the occurrence and development of the disease.

\subsection{Application of Mesenchymal Stem Cells in Alzheimer's Disease Research}

Because mesenchymal stem cells are easy to obtain and maneuverable, mesenchymal stem cells are a good treatment method. The sources of MSCs are diverse, and fatty tissue is one of the most common sources of MSCs. MSCs derived from adipose tissue can differentiate into neuron-like cells and astrocytes. Their biggest advantage is that the operation is relatively simple, and they have blood-brain barrier permeability which can be injected intravenously and cause a less immune response. The basic principle of mesenchymal stem cell therapy is to promote the differentiation of transplanted stem cells to replace damaged or dead neurons $[11,15]$.

In the latest research, it has been found that the exosomes released by mesenchymal stem cells are of great significance in the treatment of neurodegenerative diseases. The exosomes secreted by mesenchymal stem cells can be used in the treatment of neurodegenerative diseases due to their stable lipid bilayer. The transfer of biologically active molecules such as proteins, lipids, and nucleic acids carried by cells between cells participates in the communication between cells and may change the function of the recipient cells [16]. In animal models, the exosomes secreted by mesenchymal stem cells have the potential for regeneration and repair similar to 
mesenchymal stem cells. It has been confirmed that many scientists have found that mesenchymal stem cell exosomes can treat $\mathrm{AD}$ in animal models, and it is effective. For example, Katsuda et al. [17] found that adipose-derived MSCs exosomes are rich in enkephalinase, which can promote A $\beta$ degradation. We know that the accumulation of $A \beta$ and its toxicity to nerve cells are important reasons for the occurrence and progress of AD one. Studies have shown that mesenchymal stem cell exosomes help and even mediate the transplantation of mesenchymal stem cells. The mechanisms of action of mesenchymal stem cell exosomes are: 1) Cell experiments show that MSC exosomes can promote the growth of neuronal axons and dendrites and directly promote nerve repair to achieve the purpose of a nerve repair treatment. 2) MSC exosomes also have indirect nerve repair functions: MSC exosomes can interact with recipient cells, and the latter is promoted to release exosomes, which can participate in cell-to-cell communication and can also achieve nerve repair functions. 3) In the pathogenesis of $\mathrm{AD}$, the inflammatory response will also accelerate the progress of the disease. Some experiments have shown that MSC exosomes can inhibit the inflammatory response [18-19].

Because the differentiation of mesenchymal stem cells into neural cells is regulated by many factors, and other substances such as proteins in the transplantation environment may affect the differentiation of stem cells, it is difficult to differentiate mesenchymal stem cells into neural cells through cell replacement therapy. However, both the natural MSc exosomes and the modified MSCs exosomes have outstanding therapeutic effects in nervous system diseases. In the future, we can take MSc exosomes as an idea to study the treatment of extracellular vesicles. However, there are still challenges in the transformation of clinical treatment based on MSC exosomes. The exact cellular and molecular mechanisms of MSC exosomes are still unclear. The consistency and reproducibility of exosome products need to be studied and improved. In addition, the biologically active substances in MSC exosomes should be studied to clarify the specific molecular mechanisms to ensure the safety of treatment methods.

\section{Discussion}

Alzheimer's disease is a progressive central neurodegenerative disease with complicated pathogenesis. At present, FDA approved drugs for the treatment of Alzheimer's disease can only help improve the quality of life of patients, and have no obvious effect on the alleviation of the patient's disease process. There is no effective treatment method. Because stem cells have the potential to regenerate, stem cell transplantation therapy has great potential in various neurological diseases. Although people have seen the prospects of stem cell therapy in Alzheimer's disease, because the mechanism of stem cell action is not yet fully understood, stem cell clinical trials are still in the early stages. In order for stem cell transplantation technology to be successfully transformed in the clinic, it is particularly important to establish complete animal research and clinical experiment standards and pay attention to the safety, effectiveness, and ethical issues of stem cells. This article analyzes and discusses the mechanism of AD and the application of stem cell transplantation treatment. At the same time, it summarizes and analyzes the existing basic research, and puts forward some views and prospects of stem cell treatment of AD. It is hoped that stem cell transplantation can realize individualized treatment as soon as possible and bring dawn for the treatment of $\mathrm{AD}$ and other neurological diseases.

\section{Conclusion}

In summary, by analyzing the pathogenesis of $\mathrm{AD}$, and then analyzing and comparing the possible modes and approaches of neural stem cells and mesenchymal stem cells in the treatment of $\mathrm{AD}$, we can obtain that stem cell transplantation can effectively treat $\mathrm{AD}$. There are three main ways:

1. The transplanted stem cells differentiate to produce surviving neurons with normal functions, instead of damaged and dead neurons, for nerve repair

2. The paracrine pathways produced by transplanted stem cells secrete neurotrophic factors, nourish and protect existing neurons, and promote nerve growth.

3. It can also suppress inflammation through paracrine secretion of immunomodulatory factors, thereby reducing the accumulation of amyloid and slowing down the process of AD.

In future stem cell research, the above three approaches can be used to compare and judge the potential and feasibility of different stem cell treatment strategies.

\section{Acknowledgement}

First of all, I want to appreciate my university for providing me with a good learning environment and scientific research platform during my studies. I would like to express my heartfelt thanks to the teachers and professors who helped me during the university, because their series of guidance to me helped me learn a lot. I also want to thank my classmates and friends for making mutual progress in the learning process, giving me a lot of thinking and inspiration. Finally, I want to thank my beloved family members for their great support and encouragement so that I can keep moving forward.

\section{References}

1. Hong J,Jin H,Han J,et al. Infusion of human umbilical cord-derived mesenchymal stem cells effectively relieves liver cirrhosis in DNE-induced rats[J].Molecul Med Rep, 2014,9(4): 1103-1111, 2014.

2. Qian X, Hamad B, Dias-Lalcaca G. The Alzheimer disease market [J]. Nat Rev Drug Discov, 2015, 14(10): 675-676. 
3. Yao Qian, Zheng Hongyun, Li Yan. Research progress in the pathogenesis of Alzheimer's disease[J]. Occupation and Health, 2019, 35(15): 2153-2156.

4. Wang Weili, Song Cangsang. Research progress in the pathogenesis of Alzheimer's disease and clinical medication[J]. Chinese Journal of Drug Evaluation, 2019, 36(03): 204-209.

5. Chen GF,Xu TH,Yan Y,et al.Amyloid beta: structure,biology and structure-based therapeutic development $[\mathrm{J}]$.Acta Pharmacol Sin,2017,38( 9) : 1205-1235.

6. Li S,Yan Y,Jiao Y,et al. Neuroprotective Effect of Osthole on Neuron Synapses in an Alzheimer's disease Cell Model via Upregulation of MicroRNA-9 [ J ] . Journal of Molecular Neuroscience,2016,60( 1) : 71-81.

7. Yao YJ,Liang K,Jiao YN,et al. Osthole promotes differentiation into neurons and reduces neuronal apoptosis via Wnt / $\beta$-catenin signaling pathway in APP transduced neural stem cells [J].Chinese Pharmacological Bulletin,2015.

8. Simic'G,Babic'LM,Wray S,et al. Tau Protein Hyperphosphorylation and Aggregation in Alzheimer' s Disease and Other Tauopathies,and Possible Neuroprotective Strategies [J]. Biomolecules,2016,6 ( 1) : 6 .

9. Liang Zhi, Fan Linlin, Li Hongliang, Liu Jingjing, Tan Qiran, Long Shuxian, Xu Guili. Current status and new progress of drug treatment for Alzheimer's disease[J]. Southwestern National Defense Medical, 2018, 28(01): 85-87.

10. Zhang Yu, Zhao Yuming, Wang Xiaoliang, et al. Research progress and challenges of stem cell therapy for Alzheimer's disease[J]. Chinese Pharmacological Bulletin, 2015, 31(7):889-894.

11. Yang Haochi, Zhang Haiying, Zhang Yunxia, Fu Yuan. Research status of stem cell treatment of Alzheimer's disease[J]. Journal of Hainan Medical College, 2020, 26(11):876-880.

12. Lindvall O, Kokaia Z. Stem cells in human neurodegenerative disorders--time for clinical translation. J Clin Invest. 2010;120(1):29-40.

13. $\mathrm{Xu}$ Gexin, Zhang Yunxia, Zhang Haiying. The research status and development trend of stem cell therapy for Alzheimer's disease[J]. Chinese Tissue Engineering Research, 2019, 23(33): 5378-5384.

14. Ager RR, Davis JL, Agazaryan A, et al. Human neural stem cells improve cognition and promote synaptic growth in two complementary transgenic models of Alzheimer's disease and neuronal loss. Hippocampus. 2015;25(7):813-826.

15. Oh SH, Kim HN, Park HJ, et al. Mesenchymal Stem Cells Increase Hippocampal Neurogenesis and Neuronal Differentiation by Enhancing the Wnt Signaling Pathway in an Alzheimer's Disease Model. Cell Transplant. 2015;24(6):1097-1109.
16. Lener T, Gimona M, Aigner L, et al. Applying extracellular vesicles based therapeutics in clinical trials - an ISEV position paper [J]. J Extracell Vesicles, 2015, 4: 30087.

17. Katsuda T, Oki K, Ochiya T. Potential application of extracellular vesicles of human adipose tissuederived mesenchymal stem cells in Alzheimer's disease therapeutics [J]. Methods Mol Biol, 2015, 1212: 171 .

18. Wu Lingshan, Yu Zhiyuan, Luo Xiang. Research progress of mesenchymal stem cell exosomes in the treatment of neurological diseases[J]. Nerve injury and functional reconstruction, 2020, 15(11): 635-636+647.

19. Jin Shengyu, Grete, Lu Ming. Research progress of mesenchymal stem cell exosomes in the treatment of Alzheimer's disease[J]. Chinese Journal of Cell and Stem Cell (Electronic Edition), 2019, 9(04) :247-250.

20. Marsh SE, Blurton-Jones M. Prospects of neural stem cell therapy for Alzheimer's disease. In: Shetty AK, editor. Neural Stem Cells in Health and Disease. World Scientific Publishing Company; Singapore: 2015. pp. 439-465. 